\title{
SINGULARIDADES DA EDUCAÇÃO FÍSICA: UM OLHAR SOBRE A AVALIAÇÃO
}

\author{
CARACTERÍSTICAS ÚNICAS DE LA EDUCACIÓN FÍSICA: UNA MIRADA A LA \\ EVALUACIÓN
}

\section{UNIQUE FEATURES OF PHYSICAL EDUCATION: A LOOK AT EVALUATION}

\author{
Evandra Hein Mendes ${ }^{1}$ e Nilmar Defreyn ${ }^{1}$ \\ evandrahmendes@hotmail.com; nilmar-nine@hotmail.com \\ ${ }^{1}$ Universidade estadual do oeste do Paraná, Paraná, Brasil \\ Envío original: 2021-03-05 Reenviado: 2021-06-18 Aceptado: 2021-11-23 \\ Publicado: 2022-02-03
}

Doi: https://doi.org/10.15517/pensarmov.v20i1.46104

\begin{abstract}
RESUMO
A avaliação da aprendizagem está inserida em todos os componentes curriculares, inclusive na Educação Física, que aborda conhecimentos relativos à cultura corporal, como brincadeiras e jogos, ginásticas, danças, esportes, lutas e atividades de aventura. Buscando compreender melhor as características das práticas avaliativas desenvolvidas por professores de educação física, realizou-se um estudo descritivo com abordagem qualitativa, no município de Marechal Cândido Rondon, Paraná - Brasil. Os dados foram obtidos por meio da aplicação de uma entrevista semi-estruturada, que foi considerada válida, no que se refere à clareza de linguagem, a pertinência prática e a relevância teórica. A coleta de dados ocorreu entre os meses de agosto a outubro de 2017, nas dependências das escolas. Os dados foram analisados qualitativamente através da técnica de análise de conteúdo, categorizados em: funções da avaliação, quantidade de avaliações realizadas por trimestre, instrumentos/procedimentos, critérios avaliativos e dificuldades encontradas ao avaliar. Os resultados evidenciaram que o processo de avaliação se apresentou ao mesmo tempo formal e informal, realizada tanto através das provas e trabalhos, que se apresentava como uma exigência da escola e se voltava à análise da apropriação do conhecimento teórico ou conceitual, quanto por meio da observação do professor, voltada a análise da evolução motora e técnica de cada aluno, além de valorizar a participação e o envolvimento na aula. De maneira geral, a avaliação desenvolvida pelos professores buscava atender as determinações
\end{abstract}


do sistema escolar e as singularidades dos conhecimentos relativos à cultura corporal abordados nessa disciplina.

Palavras-chave: professores de educação física, avaliação escolar, Brasil.

\title{
RESUMEN
}

La evaluación del aprendizaje está incluida en todos los componentes curriculares, incluida la Educación Física, que aborda conocimientos relacionados con la cultura corporal, como juegos, gimnasia, bailes, deportes, luchas y actividades de aventura. Buscando comprender mejor las características de las prácticas evaluativas desarrolladas por profesores de educación física, se realizó un estudio descriptivo con enfoque cualitativo, en el municipio de Marechal Cândido Rondon, Paraná - Brasil. Los datos fueron obtenidos mediante de la aplicación de una entrevista semiestructurada, la cual fue considerada válida en términos de claridad de lenguaje, pertinencia práctica y pertinencia teórica. La recolección de datos ocurrió entre agosto y octubre de 2017, en las instalaciones de las escuelas. Los datos fueron analizados cualitativamente mediante la técnica de análisis de contenido, categorizados en: funciones de evaluación, número de evaluaciones realizadas por trimestre, instrumentos/procedimientos, criterios de evaluación y dificultades encontradas al evaluar. Los resultados mostraron que el proceso de evaluación fue tanto formal como informal, realizado tanto a través de pruebas como de trabajos, que se presentó como un requisito de la escuela y se centró en el análisis de la apropiación de conocimientos teóricos o conceptuales, así como mediante de la observación. del profesor, dirigidas a analizar la evolución motriz y técnica de cada alumno, además de valorar la participación e implicación en la clase. En general, la evaluación desarrollada por los docentes buscó conocer las determinaciones del sistema escolar y las singularidades de los saberes relacionados con la cultura corporal abordados en esta disciplina.

Palabras clave: profesores de educación física, evaluación escolar, Brasil.

\begin{abstract}
Learning assessment is included in all curricular components, including Physical Education, which addresses knowledge related to body culture, such as games and games, gymnastics, dances, sports, fights and adventure activities. Seeking to better understand the characteristics of evaluative practices developed by physical education teachers, a descriptive study was
\end{abstract}


carried out with a qualitative approach, in the municipality of Marechal Cândido Rondon, Paraná - Brazil. Data were obtained through the application of a semi-structured interview, which was considered valid in terms of clarity of language, practical relevance and theoretical relevance. Data collection took place between August and October 2017, on the premises of the schools. Data were analyzed qualitatively using the content analysis technique, categorized into: evaluation functions, number of evaluations performed per quarter, instruments/procedures, evaluative criteria and difficulties encountered when evaluating. The results showed that the evaluation process was both formal and informal, carried out both through tests and assignments, which was presented as a requirement of the school and focused on the analysis of the appropriation of theoretical or conceptual knowledge, as well as through the observation of the teacher, aimed at analyzing the motor and technical evolution of each student, in addition to valuing participation and involvement in the class. In general, the evaluation developed by the teachers sought to meet the determinations of the school system and the singularities of knowledge related to body culture addressed in this discipline.

Keywords: physical education teachers, scholar assessment, Brazil.

\section{INTRODUÇÃO}

No atual contexto escolar brasileiro, a Educação Física se apresenta como um componente curricular voltado ao ensino de conhecimentos construídos ao longo da existência humana, relativos à cultura corporal, materializados em práticas corporais, como brincadeiras e jogos, ginásticas, danças, esportes, lutas e atividades de aventura (Brasil, 2018).

O acesso aos saberes da cultura corporal se efetiva, geralmente, por meio de experiências vivenciais e do movimento humano, gerando uma relação singular da educação física com o saber, diferenciando-a das outras disciplinas escolares. Tal fato, promove uma inversão epistemológica na relação teoria e prática, partindo-se da prática para a teoria, evidenciada, inclusive, nas avaliações desse componente curricular (Santos et al., 2018).

Considerando as especificidades da relação teoria e prática da educação física, Darido (2012), indica que a avaliação escolar deve possibilitar ao aluno expressar seu conhecimento através de diferentes linguagens, como a corporal, a escrita e a falada.

De maneira geral, a avaliação pedagógica se constitui em um processo de recolha de informação e desenvolvimento de juízos de valor que deve considerar a diversidade de aspectos envolvidos no ensino e na aprendizagem (Onofre, 1996). Assim, a tarefa do avaliador se constitui em um permanente exercício de interpretação de sinais em diferentes 
aspectos, configurando-se, acima de tudo, como um processo de reflexão sobre e para a ação (Santos et al., 2015).

Na medida em que, for concebida como um instrumento de análise e reflexão sobre a ação, a avaliação pode subsidiar a prática pedagógica (Hoffmann, 2001). Para tanto, a avaliação deve ultrapassar o significado de exigência burocrática e articular dialeticamente teoria e prática, fornecendo pistas aos envolvidos para orientar suas ações (Santos e Maximiniano, 2013).

Além de identificar as aquisições dos alunos a avaliação pode fornecer um retrato sobre a qualidade das experiências pedagógicas e como essas tornam o processo formativo (Onofre, 1996). Assumir a avaliação formativa como parte integrante do processo contribui para a melhoria da aprendizagem, regulando o ensino e o seu planeamento, pois as informações recolhidas ao longo do período letivo, permitem identificar e estabelecer prioridades para desenvolver atividades voltadas ao desenvolvimento dos alunos (Araújo, 2017).

Ao assumir um caráter formativo na avaliação as práticas avaliativas precisam estar voltadas à orientação do ensino e melhoria da aprendizagem, valorizando o processo individual de desenvolvimento e evolução do aluno, assim como o contexto e as experiências pessoais anteriores ao processo de ensino e aprendizagem (Ramiro, 2011; Araújo, 2015).

Nesta perspectiva, a avaliação pressupõe a recolha e interpretação de dados discutidos coletivamente, entre professores e alunos, para as tomadas de decisão frente aos progressos e problemas de aprendizagem verificados, identificando novas prioridades (Carvalho, 1994). Dessa forma, a avaliação se estabelece como "o espaço ideal para mediação/aproximação/diálogo entre as alternativas de ensino do professor e os percursos de aprendizagem dos alunos" (Bonesi \& Souza, 2006, p. 135).

Para que os envolvidos no processo de ensino e aprendizagem possam compreender o potencial de utilização da avaliação na retroalimentação do ensino e na promoção da aprendizagem dos alunos, se torna imprescindível a realização de pesquisas e estudos sobre o tema, que ainda apresenta escassez de produção científica. Nesse contexto, o presente estudo tem como objetivo retratar as práticas avaliativas desenvolvidas por docentes de educação física no ensino médio. 


\section{MATERIAIS E MÉTODOS}

\section{Desenho e participantes}

A investigação se constituiu em um estudo descritivo com abordagem qualitativa e envolveu todas as escolas públicas e particulares do município de Marechal Cândido Rondon, situado no oeste do estado do Paraná - Brasil, que ofertavam o ensino médio. Com o intuito de que a amostra representasse a população de professores que ministrava aulas de educação física no ensino médio do referido município, foi convidado um professor de cada escola para participar da investigação. Todos os professores convidados aceitaram participar da pesquisa, totalizando sete docentes, que foram identificados com as siglas P1, P2, P3... etc, para garantir o seu anonimato. O grupo participante dessa investigação tinha contrato de trabalho igual ou maior que 20 horas semanais, possuía idade entre 41 a 54 anos e mais de 20 anos de experiência profissional.

\section{Instrumentos}

O instrumento utilizado para obter informações acerca das características das práticas avaliativas dos professores foi uma entrevista semi-estruturada, com as seguintes questões: "Qual é o papel de avaliação na Educação Física no Ensino Médio? Quais aspectos do desenvolvimento dos alunos são avaliados? Quantas avaliações são realizadas por trimestre? Quais instrumentos/procedimentos são utilizados para avaliar os alunos? Enfrenta alguma dificuldade ao avaliar os alunos do ensino médio? Qual?"

O roteiro da entrevista foi submetido a um processo de validação de conteúdo, a partir de três dimensões que, conforme Cassepp-Borges et al. (2010), envolvem a clareza de linguagem (compreensão dos termos e expressões textuais), a pertinência prática (grau de importância dos itens para o tema abordado) e a relevância teórica (relação com os pressupostos teóricos da área). Os itens foram analisados por um grupo de doutores em educação física, indicando a adequação de cada item por meio de uma escala do tipo Likert atribuindo um conceito que correspondia a "1 - inadequado, 2 - pouco adequado, 3 aceitável, 4 - adequado e 5 - muito adequado". Além disso, os avaliadores tinham a possibilidade de expressar suas opiniões ou sugestões em espaço abaixo de cada item, indicando alterações que julgassem necessárias. Assim, o instrumento de coleta foi considerado válido ao apresentar índice maior ou igual a $80 \%$ relativos ao percentual de concordância interobservadores. 


\section{Procedimentos}

A aplicação da entrevista ocorreu nas dependências das escolas, uma única vez, no horário de preferência do professor, entre os meses de agosto a outubro de 2017. No transcorrer da entrevista, as falas foram gravadas e posteriormente transcritas, respeitando todos os vocábulos. Em seguida, foram devolvidas aos professores, por meio de correio eletrônico, para possíveis alterações com a finalidade de validar as declarações (Negrine, 2004).

\section{Análise de dados}

Os dados das entrevistas foram analisados qualitativamente através da técnica de análise de conteúdo, que envolveu a pré-análise, a exploração do material e o tratamento dos resultados (Bardin, 2009). As respostas foram categorizadas em: funções da avaliação na Educação Física, quantidade de avaliações realizadas por trimestre, instrumentos/procedimentos de avaliação, critérios avaliativos utilizados pelos professores e dificuldades encontradas pelos professores ao avaliar.

A pesquisa foi aprovada pelo comitê de ética da Unioeste 2.195 .215 e obteve com as escolas os termos de autorização para realização da pesquisa, bem como com os professores os termos de consentimento livre e esclarecido, todos devidamente assinados pelos responsáveis.

\section{RESULTADOS}

Para melhor organização do texto, os resultados são apresentados de acordo com as seguintes categorias: funções da avaliação na Educação Física, quantidade de avaliações realizadas por trimestre, estratégias de avaliação, critérios avaliativos utilizados pelos professores e dificuldades encontradas pelos professores ao avaliar, obtidas a partir das seguintes questões: "Qual é o papel de avaliação na Educação Física no Ensino Médio? Quais aspectos do desenvolvimento dos alunos são avaliados no período letivo? Quantas avaliações são realizadas por trimestre? Quais instrumentos/procedimentos são utilizados para avaliar os alunos? Enfrenta alguma dificuldade ao avaliar os alunos do ensino médio? Qual?"

A tabela 1 evidencia a análise das funções da avaliação na educação física, que segundo os professores, pode ser utilizada tanto como método de despertar o interesse dos alunos pela aula, quanto de acompanhar o desenvolvimento ou a evolução e ainda de verificar a aquisição de conhecimento. 
Tabela 1.

Funções da avaliação na educação física segundo professores entrevistados

\begin{tabular}{lc}
\hline Funções da avaliação & Professores \\
\hline $\begin{array}{l}\text { Método de despertar interesse dos alunos pela } \\
\text { atividade física }\end{array}$ & $\mathrm{P} 1, \mathrm{P} 4, \mathrm{P} 5, \mathrm{P} 7$ \\
$\begin{array}{l}\text { Acompanhar o desenvolvimento e evolução dos alunos } \\
\text { Verificar o conhecimento adquirido }\end{array}$ & $\mathrm{P} 2, \mathrm{P} 4, \mathrm{P} 6$ \\
Exigência burocrática & $\mathrm{P} 2, \mathrm{P} 3, \mathrm{P} 7$ \\
Autoavaliação docente & $\mathrm{P} 3, \mathrm{P} 7$ \\
\hline
\end{tabular}

Fonte: elaborado pelos autores.

A tabela 2 diz respeito à quantidade de avaliações realizadas em um trimestre, demonstrando que a maioria dos professores realizavam três avaliações durante o trimestre.

Tabela 2.

Quantidade de avaliações realizadas por trimestre segundo professores entrevistados

\begin{tabular}{lc}
\hline Quantidade de avaliações & Professores \\
\hline Três avaliações & P1, P2, P4, P7 \\
Duas avaliações & P5, P6 \\
Quatro avaliações & P1, P3 \\
\hline
\end{tabular}

Fonte: elaborado pelos autores.

A tabela 3 , demonstra a diversidade de estratégias de avaliação e fontes de recolha de dados sobre a aprendizagem dos alunos, entre eles as avaliações práticas e teóricas, seminários, trabalhos e observações no decorrer das aulas. 
Tabela 3.

Estratégias de avaliação utilizadas pelos professores entrevistados

\begin{tabular}{lc}
\hline Estratégias de avaliação & Professores \\
\hline Avaliação prática & $\mathrm{P} 1, \mathrm{P} 2, \mathrm{P} 3, \mathrm{P} 4, \mathrm{P} 6, \mathrm{P} 7$ \\
Avaliação teórica & $\mathrm{P} 1, \mathrm{P} 3, \mathrm{P} 6, \mathrm{P} 7$ \\
Seminários & $\mathrm{P} 2, \mathrm{P} 3, \mathrm{P} 5, \mathrm{P} 7$ \\
Trabalhos & $\mathrm{P} 2, \mathrm{P} 4, \mathrm{P} 5, \mathrm{P} 7$ \\
Observação & $\mathrm{P} 2, \mathrm{P} 4, \mathrm{P} 6, \mathrm{P} 7$ \\
\hline
\end{tabular}

Fonte: elaborado pelos autores

A tabela 4 possibilita visualizar a análise dos critérios que os professores utilizavam para avaliar os alunos, evidenciando que o envolvimento e comportamento, o conhecimento teórico e o desenvolvimento motor eram os principais aspectos considerados no decorrer do processo avaliativo.

Tabela 4.

Critérios avaliativos utilizados pelos professores entrevistados

\begin{tabular}{lc}
\hline Critérios de avaliação & Professores \\
\hline Envolvimento e comportamento & $\mathrm{P} 1, \mathrm{P} 2, \mathrm{P} 4, \mathrm{P} 5, \mathrm{P} 6, \mathrm{P} 7$ \\
Conhecimento & $\mathrm{P} 3, \mathrm{P} 4, \mathrm{P} 6, \mathrm{P} 7$ \\
Desenvolvimento & $\mathrm{P} 2, \mathrm{P} 4, \mathrm{P} 6$ \\
\hline
\end{tabular}

Fonte: elaborado pelos autores

A tabela 5 demonstra as principais dificuldades encontradas pelos professores no decorrer do processo avaliativo, entre as quais se destacam a aplicação das avaliações práticas, a análise das condições individuais dos alunos, o descaso da disciplina e a quantidade insuficiente de aulas para realizar tal tarefa. 
Tabela 5.

Dificuldades enfrentadas pelos professores ao avaliar

\begin{tabular}{lc}
\hline Dificuldades & Professores \\
\hline Avaliação prática & $\mathrm{P} 3, \mathrm{P} 6, \mathrm{P} 7$ \\
Individualidade & $\mathrm{P} 1, \mathrm{P} 2$ \\
Descaso com a disciplina & $\mathrm{P} 4$ \\
Quantidade de aulas & $\mathrm{P} 6$ \\
\hline
\end{tabular}

Fonte: elaborado pelos autores

\section{DISCUSSÃO}

De acordo com Libâneo (1994), na educação a avaliação, cumpre pelo menos três funções: pedagógico-didática, de diagnóstico e de controle, que atuam de forma interdependente. Assim, a avaliação auxiliará no cumprimento dos objetivos gerais e específicos da educação escolar, contribuindo para a assimilação e fixação, corrigindo os erros e possibilitando o aprimoramento, desenvolvendo as suas capacidades cognitivas e evidenciado sua função social.

Conforme Silva (2010), as avaliações devem ter seus objetivos bem definidos e claros, buscando que o objeto da avaliação (aluno) consiga demonstrar seu conhecimento nos diferentes procedimentos. Além disso, Libâneo (1994), aponta que através da verificação e qualificação dos resultados obtidos, a avaliação possibilita determinar a correspondência dos objetivos propostos para orientar a tomada de decisões em relação às atividades didáticas.

Em consonância, para os professores participantes da investigação, a avaliação assume várias funções na prática pedagógica da educação física, sendo utilizada tanto como método de despertar o interesse dos alunos pela aula, quanto de acompanhar o desenvolvimento ou a evolução e ainda de verificar a aquisição de conhecimento. Isso se justifica para P4, pela necessidade de avaliar "[...] o processo de evolução do aluno, desde o momento de sua entrada na escola, e todo o processo que foi acontecendo com ele, agregando valores relacionados com a Educação Física até aquele ponto".

Além de se constituir em uma exigência burocrática da escola, a avaliação também pode ser usada como possibilidade de avaliação do desenvolvimento da prática pedagógica, como informa o P3, "[...] o objetivo da avaliação é verificar se o aluno adquiriu conhecimento e também uma forma avaliativa que é exigida pela escola, mas o maior objetivo é verificar se houve aprendizagem, e uma forma de auto avaliação docente para verificar se é preciso rever o conteúdo ou então utilizar outra metodologia". 


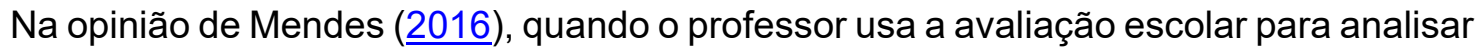
a evolução dos alunos e refletir sobre sua prática pedagógica, demonstra compreender de forma ampliada as possibilidades e contribuições da avaliação escolar.

De maneira geral, o sistema de avaliação é desenvolvido pelos professores de acordo com o que se preconiza no projeto político pedagógico da escola, que determina pesos, estratégias ou instrumentos para a coleta de dados e a distribuição no período letivo (trimestre/bimestre). No contexto pesquisado, a maioria dos professores realizava três avaliações no trimestre, o que significa que os alunos tinham mais oportunidades de demonstrar a evolução do conhecimento e os professores a possibilidade de coletar mais amostras do aprendizado para construir a nota no final de cada trimestre. Isso também reflete nas formas diferenciadas de avaliação que cada professor aplica, promovendo a igualdade de conhecimentos e respeitando as particularidades dos seus alunos, possibilitando ao aluno expressar o conhecimento de formas diferentes entre elas provas práticas, teóricas, trabalhos e seminários.

O P3 esclarece os motivos da diversificação das estratégias na seguinte fala, "[...]as avaliações que proporciono são bastante diversificadas. Se eu proporcionar somente avaliações práticas, eu não vou estar respeitando a individualidade de cada um, então com o passar dos anos eu observei que são necessários realizar avaliações escritas, orais, avaliações práticas, e não somente avaliações práticas, porque eu preciso trabalhar com as diferenças individuais, uns tem mais facilidades na fala, outras mais facilidades na escrita, outros mais na questão gestual de expressão e que eu devo desenvolver ambos e não somente um. Assim, as avaliações ficam mais condizentes com a avaliação dos demais professores e disciplinas".

Apesar da diversidade de uso de estratégias avaliativas, destacou-se o uso das avaliações práticas como a estratégia mais utilizada para avaliar os alunos nas aulas de educação física do ensino médio, pois de acordo com P1, "Na prova prática você avalia as habilidades, que são trabalhadas na educação física[...]".

Resultados semelhantes aos encontrados nessa investigação foram encontrados nos estudos de Ramiro (2011), que também identificou a utilização de provas teóricas, trabalhos, seminários e provas práticas para avaliar os alunos na educação física. Outro fato a ser destacado é a necessidade de utilização dos procedimentos elencados no Projeto Político da escola, que conforme Silva (2010) reflete a construção coletiva de um plano de intenções e ações, definindo as ações pedagógicas que possibilitem a formação do cidadão para a sociedade.

Libâneo (1994) indica a diversificação dos procedimentos e o equilíbrio entre avaliações 
quantitativas e qualitativas, pois são importantes para o desenvolvimento das potencialidades e da criatividade dos alunos e esclarece que o uso de apenas um tipo pode gerar inibição e prejudicar o crescimento pessoal. Nesse sentido, Luckesi (2010, p. 199-200) propõe que:

O entendimento correto da avaliação consiste em considerar a relação mútua entre os aspectos quantitativos e qualitativos. A escola cumpre uma função determinada socialmente, a de introduzir as crianças e jovens no mundo da cultura e do trabalho; [...] o professor deve organizar o ensino, mas o seu objetivo é o desenvolvimento autônomo e independente do aluno. Desse modo, a quantificação deve transformarse em qualificação, isto é, numa apreciação qualitativa dos resultados verificados.

Estudos diagnósticos que buscavam analisar as possibilidades pedagógicas de instrumentos avaliativos na educação física (Santos \& Maximiniano, 2013; Berni, 2010; Diniz \& Amaral, 2009) indicaram que, a diversificação amplia a compreensão do professor acerca do desenvolvimento dos alunos e a utilização de procedimentos de cunho qualitativo, como o parecer descritivo, auxiliam na formação de alunos críticos e autônomos.

A pesquisa de Santos et al. (2015), que testou a utilização de vários instrumentos para avaliar, como o diário, os desenhos e atividades escritas, identificou que a diversidade de coleta de dados sobre a aprendizagem dos alunos ampliou a perspectiva de análise do professor e a compreensão dos próprios educandos acerca das suas vivências.

Em estudo foi apontada diversas formas de realização de avaliações como provas orais, práticas orais, trabalhos e a auto avaliação na educação física escolar pelos professores (Mendes \& Barbosa-Rinaldi, 2020).

$\mathrm{Na}$ educação física, para Bratisfiche (2003) é preciso considerar todo o processo de evolução e não somente o resultado, ou seja, evitar apenas quantificar os resultados que expõem o rendimento e desempenho medido por testes físicos ou motores, mas considerar a subjetividade e o desenvolvimento no decorrer do processo, assim o aluno será o protagonista e avaliado segundo aspectos formativos.

Nota-se que os professores avaliavam o envolvimento do aluno e o comportamento, atribuindo conceito a partir da sua observação durante a aulas, como explica o P5, "uma das principais coisas que avalio em todas as aulas, é a atitude. Temos uma avaliação de atitude que são 10 questões, e eles já sabem no primeiro bimestre como acontece essa avaliação, em cima de vários aspectos: cuidado com o material, pontualidade, participação em aula, cuidado com o colega, o que acontece durante a aula mesmo. Então assim, ele já sabe como é avaliado, e não sai uma nota para eles do nada". 
Apesar da análise do envolvimento e comportamento dos alunos nas aulas ser considerado importante no processo de avaliação, os professores também avaliavam o conhecimento teórico ou conceitual dos conteúdos e o desenvolvimento motor, como esclarece o P4“[...] é feito um trabalho de um tema que seja relevante para Educação Física no Ensino Médio e que façam os alunos refletirem sobre o que é Educação Física, ou, o que compõe a Educação Física para desmistificar que Educação Física é só jogar Futsal. [...] a avaliação da participação é pontuada ao aluno, no sentido de que participar não é estar dentro da quadra. Participar é estar ativo dentro da quadra, em cada uma das propostas estimuladas a eles. A terceira avaliação, que é interesse e evolução do aluno, é que eu costumo anotar tudo o que acontece na aula, quem fez, quem não fez, e aí eu tenho parâmetros para posteriormente lançar algum conceito".

$\mathrm{Na}$ opinião de Santos et al. (2015), a avaliação escolar vem se modificando no decorrer dos últimos anos, sendo reconhecida como possibilidade de diagnosticar e valorizar a expressão individual, a cultura própria, a manifestação de afeto, como um meio para aprendizagem e formação integral do educando na medida em que analisa o desenvolvimento do aluno sob diferentes aspectos ou dimensões.

Conforme Gomes e García (2011), é preciso refletir e discutir mais sobre a avaliação na Educação Física, pois ela assume diferentes faces para atender às demandas dos currículos existentes, em que ora é caracterizada como verificação de aprendizagens obtidas e ora é vista como atividade pedagógica de reorganização didática.

Se por um lado, o processo de avaliação possibilita aos professores analisar o desenvolvimento ou aprendizagem dos alunos e refletir sobre sua atuação pedagógica, por outro exige a tomada de inúmeras decisões que são encaradas em muitos casos como dificuldades da prática docente. As principais dificuldades estavam relacionadas com as avaliações práticas e a análise da individualidade dos alunos, como indica o P3, "[...] avaliar é a coisa mais difícil na vida, porque você faz um julgamento, o que é muito difícil. Porque de repente sob meu ponto de vista, aquele aluno poderia ter dado muito mais, e ele estava dando o máximo que podia".

O descaso com a disciplina por parte de alunos e colegas que não valorizam a disciplina e trabalham de forma descompromissada também se estabeleceu como uma dificuldade enfrentada pelos professores, como informa P4, "a dificuldade que eu enfrento é receber alunos de outros colégios, sem fundamentação. A dificuldade que eu enfrento é de ter colegas da educação física, que ainda deixam o aluno fazer o que quer".

Por fim, o número insuficiente de aulas foi apontado pelos professores como outra dificuldade enfrentada, pois prejudica o desenvolvimento do aluno e a avaliação por parte do 
professor, como explica o P6, "se torna difícil avaliar porque temos uma aula por semana, então o aluno tem uma evolução mais lenta. Já quando tínhamos 3 aulas semanais, você conseguia ver um desenvolvimento muito maior e hoje com uma aula só, você sente um pouco mais de dificuldade".

\section{CONCLUSÕES}

Ao final desse estudo, realizado por meio de entrevistas com docentes de educação física no ensino médio, que envolveram questões acerca do papel da avaliação no processo de ensino, os aspectos avaliados no desenvolvimento dos alunos, a quantidade de avaliações realizadas por trimestre, os instrumentos/procedimentos utilizados e as dificuldades enfrentadas no desenvolvimento das avaliações, foi possível compreender como as práticas avaliativas se apresentam no contexto investigado.

No contexto investigado, a avaliação assumia várias funções na prática pedagógica da educação física, sendo utilizada tanto como método de despertar o interesse dos alunos pela aula, quanto de acompanhar o desenvolvimento ou a evolução e ainda de verificar a aquisição de conhecimento. Além de perceber a avaliação como uma exigência burocrática da escola, os professores indicaram o seu uso como possibilidade de avaliação da própria prática pedagógica.

Os instrumentos ou estratégias de avaliação, bem como a quantidade de avaliações realizada por trimestre atendiam ao proposto pelo sistema da escola. As provas teóricas e trabalhos eram determinadas pelo sistema avaliativo da escola para todas as disciplinas, inclusive para a educação física. Além desses instrumentos ou estratégias, os professores utilizavam ainda avaliações práticas por meio de observações em aulas, com o intuito de avaliar o aluno em ação.

O envolvimento e comportamento, o conhecimento teórico e o desenvolvimento motor dos alunos eram os principais aspectos considerados no decorrer do processo avaliativo.

As principais dificuldades enfrentadas pelos professores ao avaliar estavam associadas ao número reduzido de aulas da disciplina, a diversidade das condições dos alunos e o caráter subjetivo envolvido na avaliação.

Nesse sentido, concluímos que a avaliação realizada por professores de educação física se mostrou no contexto investigado, vinculada às determinações do sistema adotado pelas escolas, mas também direcionado às singularidades e particularidades do corpo de conhecimentos relativos à cultura corporal abordados nessa disciplina.

Esse estudo limitou-se a investigar a avaliação em um município do oeste do Paraná, assim sugere-se a realização de outras investigações com área de maior abrangência. 


\section{REFERÊNCIAS}

Araújo, F. (2015). A avaliação formativa e seu impacto na melhoria da aprendizagem [Tese Doutorado em Educação Física, Pontifícia Universidade Universidade de Lisboa]. https://www.repository.utl.pt/handle/10400.5/8344

Araújo, F. (2017). A avaliação e a gestão curricular em Educação Física-um olhar integrado. Boletim Sociedade Portuguesa de Educação Física, (32), 121-133. https://boletim.spef.pt/spef/index.php/spef/article/viewFile/227/214

Bardin, Laurence. (2009). Análise de Conteúdo. Edições 70.

Berni, K. (2010). Avaliação por parecer descritivo na educação física escolar: estudo de caso [Dissertação Mestrado, Universidade Federal de Pelotas, Brasil]. https://wp.ufpel.edu.br/ppgef/files/2014/04/katia berni.pdf

Bonesi, P., \& Souza, N. (2006). Fatores que dificultam a transformação da avaliação na escola. Estudos em Avaliação Educacional, 17(34), 129-154. http://www.fcc.org.br/pesquisa/publicacoes/eae/arquivos/1288/1288.pdf

Bratifische, S. (2003). Avaliação em educação física: um desafio. Revista da Educação Física/UEM, 14(2),

21-31. https://www.researchgate.net/publication/277037150 AVALIACAO EM EDUCACAO FISICA UM DESAFIO

Cassepp-Borges, V., Balbinotti, M.A.A. \& Teodoro, M.L.M. (2010). Tradução e validação de conteúdo: uma proposta para adaptação de instrumentos. In: L. Pasquali (Org.), Instrumentação psicológica: fundamentos e práticas. Artmed.

Carvalho, L. (1994). Avaliação das aprendizagens em Educação Física. Boletim Sociedade portuguesa de educação física, (10-11), 151. https://boletim.spef.pt/index.php/spef/article/view/163

Darido, S. (2012). A avaliação da educação física na escola. In: Conteúdos e didática de $\begin{array}{llll}\text { Educação Física } & \text { (pp.127-140). Cultura }\end{array}$ https://acervodigital.unesp.br/handle/123456789/41554?locale=pt BR

Diniz, J., \& Amaral, S. C. F. (2009). A avaliação na educação física escolar: uma comparação entre as escolas tradicional e ciclada. Revista Movimento, 15(1), 241-258. https://seer.ufrgs.br/Movimento/article/view/2085

Fernandes, S., \& Greenvile, R. (2007). Avaliação da aprendizagem na educação física escolar. Revista $\quad$ Motrivivência, 120-138. https://periodicos.ufsc.br/index.php/motrivivencia/article/view/6473

Hoffmann, J. (2001). Avaliar para promover: as setas do caminho. Mediação. 
Gomes, N., \& García, M. (2011). Avaliação da aprendizagem em educação física: uma escrita autopoiética. Estudos em Avaliação Educacional, 22(49), 285-304. http://www.fcc.org.br/pesquisa/publicacoes/eae/arquivos/1638/1638.pdf

Libâneo, J. (1994). Didática: teoria da instrução e do ensino. Cortez.

Luckesi, C. (2010). Avaliação da aprendizagem escolar (20 ed.). Cortez.

Mendes, E. H. (2016). Avaliação da aprendizagem na Educação Física Escolar: caracterização e habitus de professores do Paraná [Tese de Doutorado, Universidade

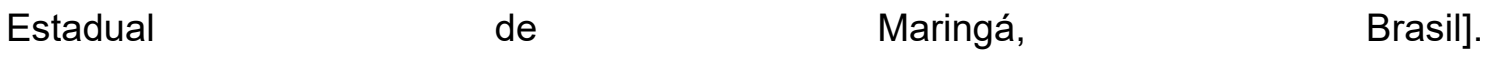
http://repositorio.uem.br:8080/jspui/bitstream/1/2149/1/000225288.pdf

Mendes, E. H. \& Barbosa-Rinaldi, I. P. (2019). Avaliação da aprendizagem na educação física escolar: caminhos percorridos e desafios atuais. Caderno de Educação Física e Esporte, 18(1), 119-123. https://erevista.unioeste.br/index.php/cadernoedfisica/article/view/23051

Minayo, M., \& Sanches, O. (1993). Quantitativo-Qualitativo: oposição ou complementaridade? Caderno de Saúde Pública, 9(3), 239-262. https://doi.org/10.1590/S0102-311X1993000300002

Ministério da Educação do Brasil. (2018). Base Nacional Comum Curricular. Brasília. http://basenacionalcomum.mec.gov.br/

Negrine, A. (2004). Instrumento de coleta de informações na pesquisa qualitativa. In: A. Triviños \& V. Molina Neto, A pesquisa qualitativa na Educação Física: alternativas metodológicas (pp.61-93). Editora da UFRGS/Sulina.

Onofre, M. (1996). Educação Física sem Avaliação: uma perversão consciente?. Boletim Sociedade Portuguesa de Educação Física, (13), 51-59. https://boletim.spef.pt/index.php/spef/article/viewFile/172/159

Ramiro, F. (2011). A Avaliação da Aprendizagem na Educação Física Escolar em Ferraz de Vasconcelos [Dissertação de Mestrado, Universidade São Judas Tadeu, Brasil]. https://www.usjt.br/biblioteca/mono disser/mono diss/2012/186.pdf

Santos, W., Mathias, B., Cassani, J., \& Vieira, A. (2015) Avaliação na educação física escolar: reconhecendo a especificidade de um componente curricular. Movimento, Revista da Escola de Educação Física da UFRGS, 21(1), 191-202. https://doi.org/10.22456/1982$\underline{8918.46895}$

Santos, W., \& Lima, F. (2013). Avaliação na educação física escolar: singularidades e diferenciações de um componente curricular. Revista Brasileira de Ciências do Esporte, 35(4), 883-896. https://doi.org/10.1590/S0101-32892013000400006 
Santos, W., Maximiano, F., \& Frossard, M. (2018). Narrativas docentes sobre avaliação do ensino-aprendizagem: da formação inicial ao contexto de atuação profissional. In W. Santos (Org.), Avaliação na educação física: diálogos com a formação inicial do Brasil. Appris.

Silva, J. (2010). Métodos de avaliação em Educação Física no ensino fundamental [Tese Doutorado, Universidade Estadual de Campinas, Brasil]. http://bdtd.ibict.br/vufind/Record/CAMP ca38cacff9f7ac9b314120425d8f063a 